\title{
High-Efficiency Biocatalytic Conversion of Thebaine to Codeine
}

\author{
Xu Li, Konrad Krysiak-Baltyn, Luke Richards, Ailsa Jarrold, Geoffrey W. Stevens, Tim Bowser, \\ Robert E. Speight, and Sally L. Gras*
}

Cite This: ACS Omega 2020, 5, 9339-9347

Read Online

\section{ACCESS | Lill Metrics \& More | 回 Article Recommendations | S1 Supporting Information}

ABSTRACT: An enzymatic biosynthesis approach is described for codeine, Thebaine the most widely used medicinal opiate, providing a more environmentally sustainable alternative to current chemical conversion, with yields and productivity compatible with industrial production. Escherichia coli strains were engineered to express key enzymes from poppy, including the recently discovered neopinone isomerase, producing codeine from thebaine. We show that compartmentalization of these enzymes in different cells is an effective strategy that allows active spatial and temporal control of reactions, increasing yield and volumetric productivity and reducing byproduct generation. Codeine is produced at a yield of $64 \%$ and a volumetric productivity of $0.19 \mathrm{~g} /(\mathrm{L} \cdot \mathrm{h})$, providing the basis for an industrially applicable aqueous

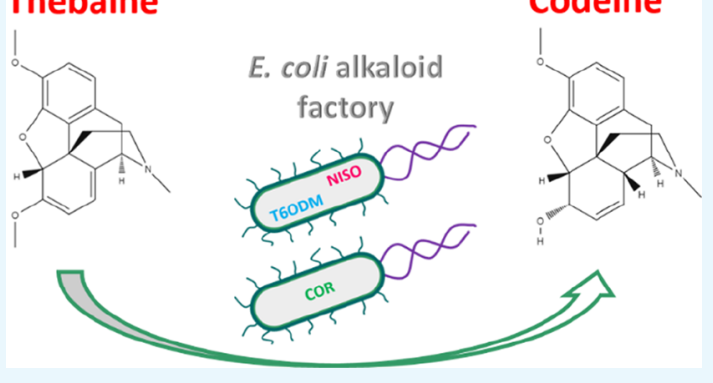
whole-cell biotransformation process. This approach could be used to redirect thebaine-rich feedstocks arising from the U.S. reduction of opioid manufacturing quotas or applied to enable total biosynthesis and may have broader applicability to other medicinal plant compounds.

\section{INTRODUCTION}

Enzymatic biotransformation offers a new route to the production of medicinal plant products, which is potentially more environmentally sustainable than current chemical conversion processes. The development of processes with high yields and productivities compatible with industrial production, however, remains a challenge for many plant products. Codeine, the world's most widely used opiate, is a relevant example for enzymatic biotransformation, with potential for rapid industrial uptake. Currently, 85-90\% of codeine is produced by methylation of morphine, an alkaloid generally more abundant in poppy than codeine, ${ }^{1}$ using large volumes of organic solvents, a toxic methylating agent and producing a carcinogenic byproduct; the downstream purification processes are also complex. While codeine-rich poppy genetic variants ${ }^{1,2}$ have been developed, this approach still carries climatic risk and requires optimization of extraction yield. Direct production of codeine from thebaine could also have significant impact following the reduction in manufacturing quota for opioids in $2018^{3}$ by the Department of Justice and U.S. Drug Enforcement Administration. This quota includes oxycodone and hydroxycodone, ${ }^{3}$ which are manufactured from thebaine, ${ }^{4}$ and the thebaine feedstock could be redirected to produce codeine, the WHO-listed essential medicine ${ }^{5}$ consumed at $>300$ ton scale annually. ${ }^{6}$ Thebaine is a natural plant precursor with lower potential for illicit use ${ }^{7}$ than morphine. A high yielding and cost-effective biosynthetic route for codeine production would therefore be a significant advance for the pharmaceutical industry.
Over the last decade, a series of studies have improved our understanding of the key biosynthetic pathways of benzylisoquinoline alkaloids occurring in planta and have investigated the construction of select pathways in microbes. ${ }^{7-19}$ The microbial production of alkaloids, such as thebaine ${ }^{7,16}$ and noscapine, ${ }^{19}$ from simple sugars or glycerol offers an alternative to chemical synthesis or the extraction of alkaloids from opium poppies. A flexible approach, however, involving multiple steps in several microbes or a combination of extraction, synthetic conversion, and/or synthesis may offer the best yield, similar to the production of artemisinic acid by synthetic biology. ${ }^{20}$

For the production of codeine, an efficient synthetic process from thebaine will be key. This will be true for conversion from thebaine-rich feedstocks or from simple carbon sources. The use of Escherichia coli to increase the thebaine yield from glycerol by $300-$ fold $^{16}$ compared to that of yeast ${ }^{7,16}$ is already a significant step toward the realization of microbial production of codeine from simple carbon sources, with further advances now needed in the conversion of thebaine to codeine.

An opportunity to improve synthetic conversion of thebaine to codeine has recently arisen from the discovery that thebaine is converted to codeine via a three-step process catalyzed by thebaine 6-O-demethylase (T6ODM), neopinone isomerase

Received: January 21, 2020

Accepted: March 23, 2020

Published: April 3, 2020 


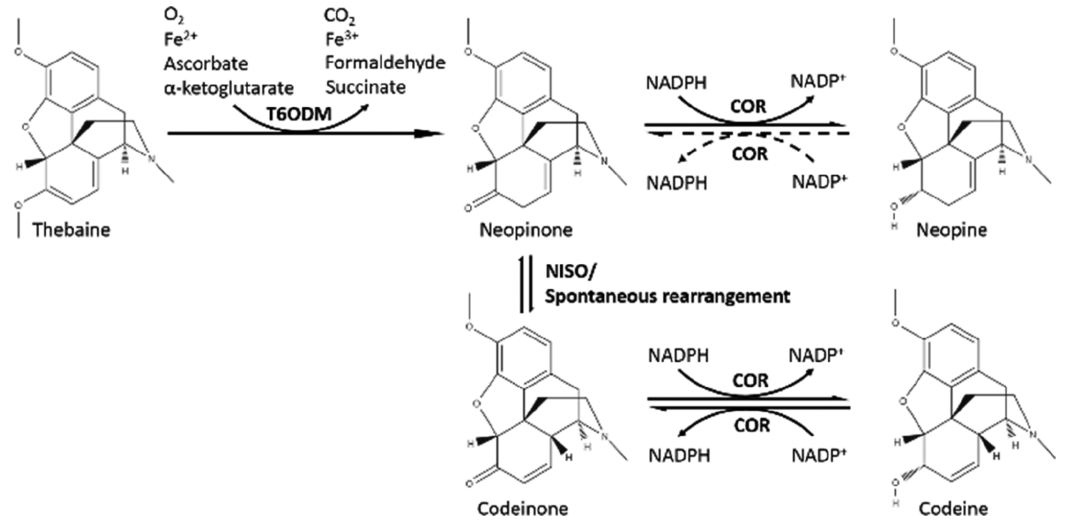

Figure 1. In vitro enzyme-catalyzed reaction of thebaine to codeine (and byproduct neopine). The first step involves the irreversible conversion of thebaine to neopinone catalyzed by T6ODM, ${ }^{9,24}$ with cosubstrates $\alpha$-ketoglutarate and oxygen and cofactors Fe(II) and ascorbate. ${ }^{9,25}$ Neopinone is then isomerized to codeinone, a reversible step ${ }^{10}$ that occurs spontaneously in aqueous solution, reaching an equilibrium state depending on solution parameters such as $\mathrm{pH}^{21}$ and potentially ionic strength or temperature. The rate at which this equilibration is achieved can be accelerated by introducing the enzyme NISO. ${ }^{10}$ The enzyme COR accepts both codeinone and neopinone as a substrate, leading to the generation of codeine or the undesired byproduct neopine together with the consumption of NADPH. ${ }^{26}$ The neopinone to neopine conversion has been reported as either an irreversible reaction ${ }^{22}$ or a reversible reaction; ${ }^{11}$ the dashed lines indicate this uncertainty.

(NISO), and codeinone reductase (COR), ${ }^{9,10}$ whereas the conversion between intermediates neopinone and codeinone, previously thought to be spontaneous, ${ }^{21}$ was shown to be catalyzed by NISO. ${ }^{10}$ The plant appears to use NISO to reduce the formation of neopine, an unwanted byproduct that occurs in vitro from the action of COR on neopinone. This process occurs in addition to the action of COR on the desired intermediate codeinone (Figure 1). The discovery of NISO, therefore, provides an opportunity to improve the biosynthetic production of codeine in a microbial system by reducing the formation of neopine.

A significant challenge for synthetic conversion, however, is the loss of spatial and temporal control that occurs when the genes encoding for key enzymes are expressed in a single microbe without the mechanisms employed by the plant to control conversion. Consequently, enzymatic biosynthesis of codeine from thebaine has typically featured low substrate conversion and low product yields (e.g., purified T6ODM, NISO, and COR converted only $0.25 \%$ of thebaine into codeine $\left.{ }^{10}\right)$. The subcellular compartmentalization of T6ODM and COR in Saccharomyces cerevisiae increased product specificity by targeting COR to an organelle and keeping T6ODM in the cytoplasm, allowing some time for rearrangement of neopinone, but only $1.8 \%$ of thebaine was converted to codeine by this technique, ${ }^{11}$ and this approach does not allow active control of the timing between reactions. The highest volumetric productivity of codeine in these systems was just $\sim 0.0006 \mathrm{~g} /(\mathrm{L} \cdot \mathrm{h})$, in part due to long reaction times and the production of neopine. ${ }^{11,22,23}$

The low substrate conversion and low time space yield illustrate the need to produce a high yielding system that can convert thebaine to codeine although being both flexible and scalable. Here, we used a robust engineered $E$. coli biocatalyst system incorporating an innovative cell-based enzyme compartmentalization strategy to allow active spatial and temporal control of the whole cell biotransformation of thebaine to codeine. By expressing T6ODM and COR in E. coli, we achieved a volumetric productivity of $0.10 \mathrm{~g} /(\mathrm{L} \cdot \mathrm{h})$, corresponding to an at least $\sim 160$-fold increase from other reported systems. ${ }^{11,22}$ The codeine yield improved from 19 to $48 \%$ with the cell compartmentalization strategy. By introducing NISO into the cell compartmentalization strategy, the yield was further improved to $64 \%$, compared to the reported $1.8 \%$ conversion of thebaine to codeine in yeast. ${ }^{11}$ The molar ratio of codeine to neopine also increased from 20:80 to $74: 26$ and the volumetric productivity was as high as $0.19 \mathrm{~g} /(\mathrm{L} \cdot \mathrm{h})$, greater than the productivities reported for other pharmaceutical biotransformations, such as paclitaxel from plant-derived intermediates or lamivudine [both at $0.1 \mathrm{~g} /(\mathrm{L}$. h)], ${ }^{27}$ illustrating the significant potential of this biocatalytic conversion system.

\section{RESULTS}

Selection of COR Isoform and Host Cell Type for Biotransformation. To date, COR isoforms from Papaver somniferum have been used to produce codeine using either purified enzyme preparations or enzymes expressed in yeast. ${ }^{11,22}$ The concentration of the undesired neopine byproduct varies between these isoforms. PsCOR1-3 produced neopine at levels similar to codeine when incorporated with T6ODM in yeast, ${ }^{22}$ while COR-B produced 5 times more neopine than codeine in yeast. ${ }^{22}$

Other COR isoforms may potentially be used to produce codeine and neopine with different levels of specificity, as COR is expressed in several species within the Papaver genera, including Papaver bracteatum, Papaver orientale, and Papaver rhoeas (Figure 2 and see Figure S2 for isoform comparison in amino acid sequences). ${ }^{28}$ In particular, isoforms from $P$. bracteatum (PbCOR) have the least sequence identity to PsCOR isoforms from $P$. somniferum ${ }^{29}$ in phylogenetic analysis (92\% identity to PsCOR-B and 90\% identity to PsCOR1-3). PbCOR1-1 was selected as a novel isoform for the biotransformations that may display altered specificity for neopine and codeine formation. PbCOR1-1 was used together with T6ODM and NISO from P. somniferum for thebaine demethylation and neopinone to codeinone isomerization, respectively.

E. coli cells were selected for biotransformation because of their recognized high capacity for protein expression and industrial applicability. ${ }^{30}$ E. coli cells were also demonstrated as an efficient platform for thebaine biosynthesis from glycerol compared with a yeast system. ${ }^{16} \mathrm{~A}$ whole cell biotransforma- 


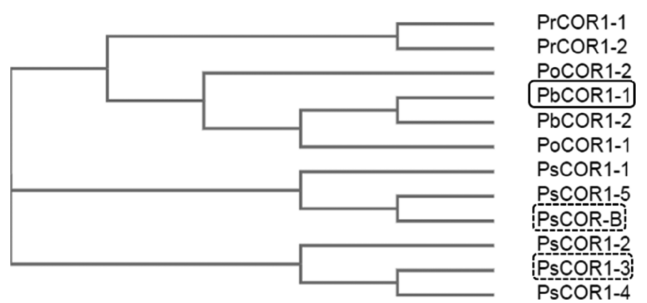

Figure 2. Phylogenetic analysis of the PbCOR1-1 isoform from $P$. bracteatum $(\mathrm{Pb})$ used in this study (highlighted with a solid rectangle) and other isoforms from $\mathrm{Pb}$ and other species [P. somniferum (Ps), P. rhoeas ( $\mathrm{Pr})$, and $P$. orientale $(\mathrm{Po})]$. The sequence of the PbCOR1-1 isoform used in this study has $92 \%$ similarity with PsCOR-B and $90 \%$ similarity with PsCOR1-3 (both highlighted with dashed rectangles), COR isoforms that have been used previously ${ }^{22}$ to catalyze codeinone to codeine (and neopinone to neopine).

tion process employing resting cells was also chosen so that the cofactors and cosubstrates, $\mathrm{Fe}^{2+}$, ascorbate, and $\alpha$-ketoglutarate for T6ODM catalysis and NADPH for COR catalysis, ${ }^{31,32}$ would be supplied by the cell, reducing process costs.

Strategy 1 -Single Cell E. coli Coexpression of T6ODM and COR for Codeine Synthesis from Thebaine. In the first biotransformation strategy (Figure 3a), we designed

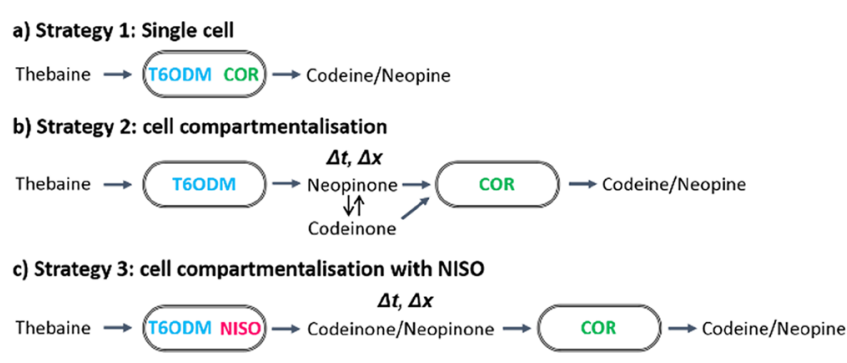

Figure 3. Three strategies for the biotransformation of thebaine to codeine (and byproduct neopine) using engineered E. coli cells. In strategy 1, shown in (a), E. coli cells are engineered to express T6ODM and COR within the same cell that converts thebaine to codeine (and neopine) with the T6ODM conversion of thebaine to neopinone, the equilibration of neopinone to codeinone, and the COR-catalyzed conversion of codeinone to codeine (and neopinone to neopine) all occurring within the same cell. In strategy 2 , shown in (b), the reactions catalyzed by T6ODM and COR are separated in different E. coli cells. The T6ODM-expressing cells convert thebaine to neopinone, while the COR-expressing cells convert codeinone to codeine or neopinone to neopine. The two cell types may be mixed simultaneously at the start of the reaction $(\Delta t=0 \mathrm{~min})$ or the addition of COR cells delayed, allowing time for neopinone to equilibrate to codeinone (in this case, $\Delta t=15,30,45,60$, or 120 $\mathrm{min})$. This strategy also allows spatial separation $(\Delta x)$ of the two cell types. In strategy 3 , shown in (c), enzymes T6ODM and NISO are contained within a single cell, and similar to strategy 2 , the reaction steps are separated in two types of E. coli cells. The addition of NISO enzyme expression is expected to catalyze neopinone conversion to codeinone in order to generate more codeine and less neopine. This strategy also allows both time and spatial separation to the reactions.

a single E. coli strain containing the plasmid pETDuetPsT6ODM-PbCOR1-1 (Figure S3) that expresses both T6ODM and PbCOR1-1, without NISO, where the threestep biosynthetic pathway from thebaine to codeine occurs within a single cell. The conversion efficiency was assessed by the biotransformation of thebaine $(1 \mathrm{mM})$ using cells in a buffered solution. Typically, thebaine was completely consumed within $35 \mathrm{~min}$ and high concentrations of products codeine and neopine were produced by $40 \mathrm{~min}$ (Figure S8), as shown by the liquid chromatography (LC) chromatograms at the start (Figure 4a; thebaine substrate only) and at the end of the reaction (Figure $4 \mathrm{~b}$; showing neopine and codeine products).

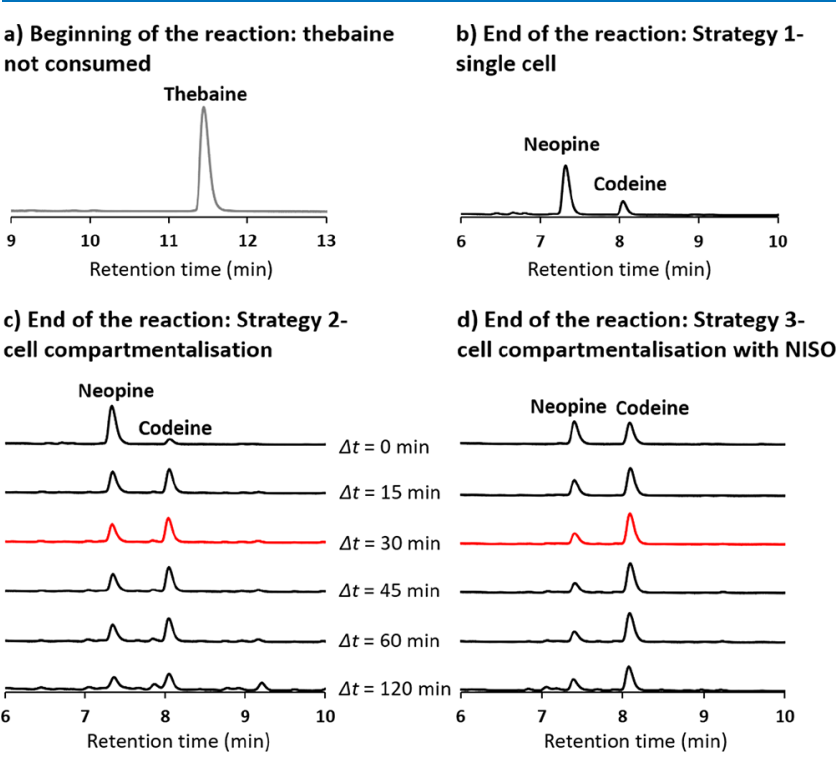

Figure 4. Conversion of thebaine to codeine and byproduct neopine using the three strategies (see Figure 3). (a) The HPLC chromatogram shows the presence of thebaine (retention time RT of $11.4 \mathrm{~min}$ ) at the start of the reaction. (b) Using strategy 1, thebaine was converted to codeine (RT of $8.1 \mathrm{~min}$ ) and neopine (RT of $7.3 \mathrm{~min}$ ) at the end of the reaction. (c) Using strategy 2, thebaine was converted to codeine and neopine, with the ratio of codeine and neopine varying as a function of the delay in COR cell addition. (d) Using strategy 3, thebaine was converted to codeine and neopine with the ratio of codeine to neopine varying as a function of the delay in COR cell addition. The chromatograms in red show the optimal conditions to achieve maximum volumetric productivity of codeine. The chromatograms are representative of three independent biological replicates.

The yield of codeine was $\sim 19 \%(0.19 \pm 0.06 \mathrm{mM})$ and neopine $\sim 75 \%(0.75 \pm 0.01 \mathrm{mM})$, corresponding to an average ratio of codeine/neopine of $20: 80$, with $\sim 94 \%$ of thebaine being consumed (Figure 5a). Under these conditions, the neopinone generated inside the cell may not have sufficient time to equilibrate with codeinone, resulting in a low concentration of codeinone and consequently a relatively low concentration of codeine and high concentration of neopine from the activity of COR on neopinone. At least 30 min was required for neopinone and codeinone to reach equilibrium when spontaneous isomerization was tested in vitro in phosphate buffer at $7.0,^{21}$ with the equilibrium ratio of codeinone/neopinone reported to range from $42: 58$ to $66: 34$ between $\mathrm{pH} 6.0$ and 8.0. The low product ratio of $20: 80$ codeine to neopine measured for the single-cell strategy here, where the cytoplasmic $\mathrm{pH}$ is expected to be $7.4-7.8,{ }^{33}$ together with the similar timescales between conversion to codeine and in vitro equilibration $(\sim 40 \mathrm{vs} \sim 30 \mathrm{~min})$ indicated that further optimization was required to increase the ratio of codeinone/neopinone available to the COR enzyme under these conditions.

In planta, only minute amounts of neopine form in the latex of opium poppy, ${ }^{22}$ in contrast to the high concentrations observed in vitro. The mechanisms employed by the plant to 

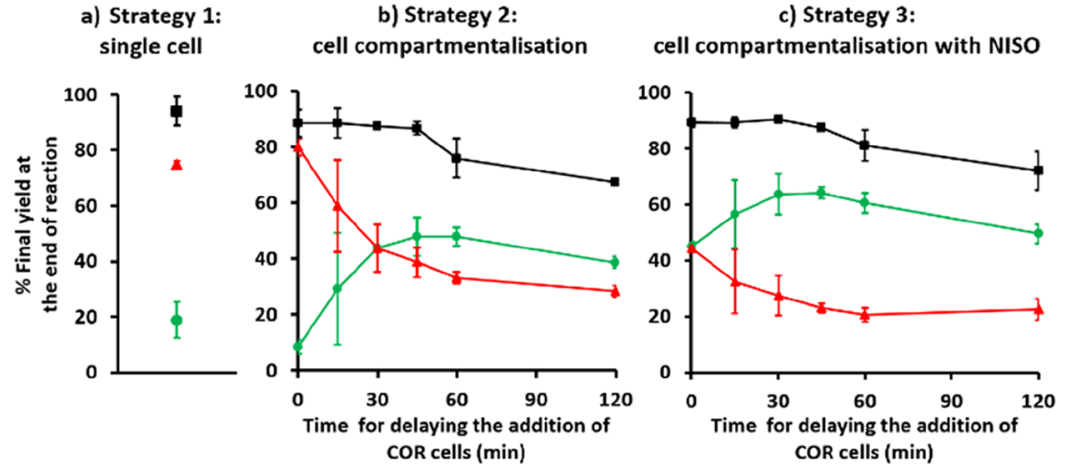

Figure 5. Yield of codeine (green $\boldsymbol{\bullet}$ ) and byproduct neopine (red $\boldsymbol{\Delta}$ ) and total yield of both opiates (black $\boldsymbol{\square}$ ) expressed as a percentage for the three biotransformation strategies. Data are shown for three biological replicates, and error bars indicate standard deviation. (a) With strategy 1 , the average yield is $19 \%$ codeine, $75 \%$ neopine, and $94 \%$ for both opiates. (b) When strategy 2 is applied, the final yield of codeine and neopine and the total yield of both opiates are a function of the delay in COR cell addition. A maximum codeine yield of $48 \%$ is achieved when COR cell addition is delayed for 45 or $60 \mathrm{~min}$. (c) When strategy 3 is applied, the final yield of codeine and neopine and the total yield of both opiates are also a function of the delay in COR cell addition. A maximum codeine yield of $64 \%$ is achieved when cell addition is delayed for 30 or 45 min.


Figure 6. Comparison of (a) codeine yield (\%) and (b) predicted time/space codeine yield [or volumetric productivity, g/(L·h)] between the three biotransformation strategies reported here $(\mathrm{S} 1-\mathrm{S} 3)$ and previously reported biotransformation studies $(1-3)$. In these studies, codeine was produced from thebaine using the following: 1 -yeast strains expressing chromosomally integrated T6ODM and PsCOR $1-3{ }^{11} 2-$ purified $^{2}$ enzymes of T6ODM, NISO, and PsCOR-B; ${ }^{10} 3$-yeast strains containing transiently expressed constructs containing T6ODM, NISO, and PsCOR-B; ${ }^{10} \mathrm{~S} 1$ - using the single-cell strategy; S2-using the cell compartmentalization strategy; and S3-using cell compartmentalization with the NISO strategy. ${ }^{*}$ Codeine yield of $0.25 \%$ is too low to be shown on the chart. $* *$ Codeine yield was not available in this prior work. ${ }^{10}$ *** Maximal possible time/space yield is therefore predicted based on a $100 \%$ yield of codeine for this prior study. The codeine time/space yield predictions for the three strategies in this study use the actual time taken for biotransformation from thebaine to codeine, which does not include the bacterial cell cultivation time, which is separated in this process within E. coli. For studies 1 and 3, the time reported in the literature may include the yeast culture time, as the duration of the biotransformation was not specified; these estimates may therefore be an underestimation of predicted time/space yield. Data for S1-3 in this study are three biological replicates. The optimal $\Delta t$ is 45 min for strategy 2 to achieve the highest codeine yield and $30 \mathrm{~min}$ for strategy 3 . The optimal $\Delta t$ is $30 \mathrm{~min}$ for strategy 2 and strategy 3 to achieve the highest codeine volumetric productivity.

avoid neopine formation have not been unraveled, but metabolic channelling ${ }^{34}$ may prevent neopine production, suggesting that strategies that can spatially and temporally control the conversion of intermediate neopinone to codeinone may more effectively produce codeine by minimizing the flux to byproduct neopine.

Strategy 2-Cell Compartmentalization for Spatial and Temporal Control of Codeine Biosynthesis in $E$. coli. A cell compartmentalization strategy (Figure $3 b$ ) was next developed to provide active temporal control of the three reaction steps, where the two enzymatically catalyzed steps were separated into two different E. coli cells. These two cell types may then be introduced at different time points during a batch reaction and the time interval can be effectively controlled; the reactions could also potentially be spatially separated in two different reactors, allowing a range of flexible reactor configurations, ${ }^{35}$ for example, in two separate sequential batch reactors or in subsequent sections of a continuous reactor. This approach may be used to provide sufficient time (theoretically $\approx 30 \mathrm{~min}$ ) to obtain the maximal concentration of codeinone using the spontaneous equilibrium from neopinone, potentially increasing the codeine yield. Two cell types were obtained by introducing either plasmid pACYC-PsT6ODM or pET28a-PbCOR1-1.

In this strategy, thebaine was introduced to T6ODM containing cells to generate neopinone for specific time intervals $(\Delta t)$ to allow the spontaneous conversion of neopinone to codeinone. Then the COR-containing cells were added to catalyze the reduction of codeinone to codeine (and the side reaction from neopinone to neopine). The time interval for delaying the addition of COR-containing cells $(\Delta t)$ was systematically varied $(0,15,30,45,60$, and $120 \mathrm{~min})$ to determine the optimal $\Delta t$ for whole cell biotransformation.

A maximum codeine yield of $\sim 48 \%(0.48 \pm 0.07 \mathrm{mM})$ was achieved when $\Delta t$ was $45 \mathrm{~min}$ (Figures $4 \mathrm{c}$ and $5 \mathrm{~b}$ ), and a similar codeine yield of $0.48 \pm 0.03 \mathrm{mM}$ was achieved when $\Delta t$ was $60 \mathrm{~min}$. This yield is 6 -fold higher than when CORcontaining cells are added at the same time as T6ODMcontaining cells $(\Delta t=0 \mathrm{~min})$ generating $\sim 8 \%$ codeine $(0.08 \pm$ $0.02 \mathrm{mM}$ ) and 2.5-fold higher compared to when the single- 
cell strategy was employed producing $\sim 19 \%$ codeine $(0.19 \pm$ $0.06 \mathrm{mM}$ ).

The codeine yield of $48 \%$ obtained using the cell compartmentalization strategy with delayed COR addition is significantly higher than previous studies using yeast strains expressing subcellularly compartmentalized T6ODM and PsCOR enzymes, ${ }^{10,11}$ where the highest codeine yield was $1.8 \%$ (Figure 6a). This comparison indicates that the cell compartmentalization strategy can effectively convert thebaine, while the optimized delay allows sufficient time for neopinone to reach equilibrium with codeinone before COR addition, resulting in a substantial amount of codeine product.

Under these optimal conditions, $39 \%(\Delta t=45 \mathrm{~min})$ or $33 \%$ $(\Delta t=60 \mathrm{~min})$ of thebaine was converted to the undesired product neopine, corresponding to a codeine to neopine ratio of 55:45 $(\Delta t=45 \mathrm{~min})$ or 59:41 $(\Delta t=60 \mathrm{~min})$, higher than the ratio of 20:80 obtained when the single-cell strategy was applied. This ratio indicates that an equilibrium between codeinone and neopinone has been achieved before COR addition that is within the range reported for spontaneous rearrangement in vitro $(42: 58$ to $66: 34){ }^{21}$ with the COR enzyme which then converts this codeinone to codeine.

Longer delays in COR addition $(\Delta t=120 \mathrm{~min})$ led to a decrease in codeine yield (39\%), possibly because of the degradation of opioids, in particular neopinone, ${ }^{24}$ which occurs after extended incubation. This degradation can be seen in the significant decrease of $33 \pm 1 \%$ in overall opiate yield and the appearance of additional peaks in the high-performance LC (HPLC) trace when COR cells are added after $120 \mathrm{~min}$ (Figures $4 c$ and $5 b$ ). These data illustrate how the optimal timescales for biotransformation involve a balance between the production of codeinone by equilibration with neopinone and the stability of the alkaloid mixture in solution; noting that these opiates can be protected from further degradation by subsequent organic extraction at the optimal point of the reaction progress. Using the cell compartmentalization strategy, the volumetric productivity (i.e., time/space yield) of codeine was $0.12 \pm 0.01 \mathrm{~g} /(\mathrm{L} \cdot \mathrm{h})$ and $0.10 \pm 0.01 \mathrm{~g} /(\mathrm{L} \cdot \mathrm{h})$ when COR addition was delayed for 45 and $60 \mathrm{~min}$, respectively. The highest volumetric productivity of codeine $(0.15 \pm 0.01 \mathrm{~g} /(\mathrm{L} \cdot \mathrm{h}))$ was achieved when COR addition was delayed for $30 \mathrm{~min}$ (Figure $6 \mathrm{~b}$ ) because the shorter $\Delta t$ reduces the time required to generate codeine. This measure of volumetric productivity is important for industrial application and should be considered when evaluating the optimal strategy for biotransformation, as both the final yield and reaction time affect productivity.

These studies demonstrate that cell compartmentalization could be used as an active strategy to significantly increase the codeine yield, although neopine formation was still a major consideration.

Strategy 3-Cell Compartmentalization with NISO To Further Improve the Codeine Yield. The recent discovery of NISO refined the pathway from neopinone to codeinone in the poppy, as this was previously presumed to occur only by nonenzyme-catalyzed equilibration. In an engineered microbial system, NISO was shown to increase the yield of codeine compared to that of neopine. ${ }^{10}$ We therefore incorporated this newly found enzyme to form a third compartmentalization strategy (Figure 3c), in an attempt to further increase the yield and volumetric productivity of codeine formation.

The design used a cell where enzymes T6ODM and NISO were coexpressed (Figure 3c) to ensure close proximity between the neopinone product generated by T6ODM catalysis and the enzyme NISO. This design was expected to catalyze the conversion of neopinone to codeinone within the same cell once the neopinone has been formed from thebaine. A second cell type containing COR, allowing temporal and spatial control, was expected to allow further opportunity for the neopinone-codeinone equilibrium to establish prior to COR-catalyzed reduction of neopinone and codeinone.

The codeine yield increased to $\sim 45 \%(0.45 \pm 0.01 \mathrm{mM})$ employing the cell compartmentalization strategy with NISO with $\Delta t=0 \mathrm{~min}$ (Figure $5 \mathrm{c}$ ), compared to $\sim 8 \%(0.08 \pm 0.02$ $\mathrm{mM}$ ) employing strategy 2 . The codeine to neopine ratio was also improved to 50:50 from 9:91 under these conditions, suggesting that the addition of NISO reduced the production of neopine and dramatically increased codeine in the final product, consistent with recent reports for this enzyme. ${ }^{10}$

The yield of codeine was further improved by the delayed addition of COR cells (Figure $5 \mathrm{c}$ ), with both $\Delta t$ of 30 and 45 min found to be optimal to achieve a maximum average codeine yield of $64 \%(0.64 \mathrm{mM})$, higher than the average yield of $48 \%(0.48 \mathrm{mM})$ achieved under optimal conditions in strategy 2 ( $\Delta t$ of 45 and $60 \mathrm{~min}$ ). In strategy 3, the average ratio of codeine to neopine was found to be 70:30 when $\Delta t$ was set as $30 \mathrm{~min}$ and 74:26 when $\Delta t$ was set as $45 \mathrm{~min}$, both higher than the optimal 59:41 in strategy 2 when $\Delta t$ was set as $60 \mathrm{~min}$ (or 55:45 in strategy 2 when $\Delta t=45 \mathrm{~min}$ ).

A recently published study using yeast for the conversion of thebaine to codeine ${ }^{10}$ provides an opportunity to compare $E$. coli and Saccharomyces cerevisae as biotransformation host strains. Using the $S$. cerevisae strain CEN.PK expressing T6ODM, NISO, and PsCOR-B and low concentrations of thebaine $(0.1 \mathrm{mM})$, neopine was not detected in the final product, indicating a high specificity of codeine production. While the percentage of thebaine converted to codeine was not stated, preventing direct comparison, the codeine yield of 0.3 $\mathrm{mg} / \mathrm{L} / \mathrm{OD}$ was 67 -fold less than the codeine yield of $19.2 \pm$ $0.6 \mathrm{mg} / \mathrm{L} / \mathrm{OD}$ in this study $(\Delta t=30$ and $45 \mathrm{~min})$. Using a dry cell weight to $\mathrm{OD}_{600}$ conversion $(0.47 \mathrm{~g}$ (dry cell)/L for E. coli BL21(DE3) $\operatorname{strain}^{36}$ and $0.53 \mathrm{~g}$ (dry cell)/L for S. cerevisae TMB3001 strain $^{37}$ (a derivative of CEN.PK) for an $\mathrm{OD}_{600}$ of (1), this leads to a codeine yield of $0.6 \mathrm{mg} / \mathrm{g}$ cell for $S$. cerevisiae cells and $40.9 \mathrm{mg} / \mathrm{g}$ cell for E. coli cells, suggesting that for this particular bioconversion, E. coli is able to achieve a greater thebaine to codeine conversion than yeast.

The incorporation of NISO also improved the volumetric productivity to $0.19 \pm 0.02 \mathrm{~g} /(\mathrm{L} \cdot \mathrm{h})$ (at $\Delta t=30 \mathrm{~min}$ ) in strategy 3 (Figure 6b) compared to $0.15 \pm 0.01 \mathrm{~g} /(\mathrm{L} \cdot \mathrm{h})$ (at $\Delta t$ $=30 \mathrm{~min}$ ) in strategy 2 .

\section{DISCUSSION}

Several studies have investigated the biotransformation of thebaine to codeine in vitro, mimicking the conversion that occurs within the poppy plant. Neopine is a common dominant byproduct in these reactions because of the undesirable action of COR on neopinone. ${ }^{22}$ In studies using cell suspension cultures of $P$. somniferum, only neopine was formed when thebaine was supplied as a substrate. ${ }^{23}$ The first study that used a microbial factory to convert thebaine (1 $\mathrm{mM}$ ) to morphine (with codeine as the intermediate product) achieved a final molar yield of $0.018 \mathrm{mM}$ codeine and 0.077 $\mathrm{mM}$ neopine, employing a yeast strain (expressing PsT6ODM and PsCOR1.3). ${ }^{11}$ This yield corresponds to a codeine/ neopine ratio of $19: 81$, with $1.8 \%$ of thebaine being converted 
into codeine. Similar ratios of 15:85 codeine/neopine have been reported using a COR enzyme sourced from the Bea's choice chemotype. ${ }^{22}$ The production of a substantial concentration of neopine in these studies could be due to the undesired intermediate neopinone having insufficient time to convert to desired intermediate codeinone, resulting in a low concentration of codeinone and consequently a low concentration of codeine and high concentration of neopine. These studies clearly illustrate that further improvements are needed to optimize the yield of codeine and ratio of codeine/ neopine in order to develop an industrially relevant process and that reaction conditions in engineered microbes are clearly very different from that of poppy where neopine is not observed.

The discovery of NISO significantly increased the ratio of codeine/neopine produced in the bioconversion of thebaine. In previous work, however, only $0.25 \mu \mathrm{M}$ of codeine was produced from $100 \mu \mathrm{M}$ of thebaine using the purified T6ODM, NISO, and COR-B in a $2 \mathrm{~h}$ assay, corresponding to a $0.25 \%$ codeine yield in $2 \mathrm{~h}^{10}$ and a potential volumetric productivity of $0.00004 \mathrm{~g} /(\mathrm{L} \cdot \mathrm{h})$. This study illustrated how the enzyme NISO can be employed to improve the codeine/ neopine ratio, but there remains a need to optimize both biotransformation yield and productivity for industrial applications.

In this work, we described three strategies to realize a whole cell biotransformation from thebaine to codeine in $E$. coli. While yeast cells are often used for the production of alkaloids from simple sugars, bacterial cells, such as E. coli, offer advantages including rapid growth, easy genetic manipulation, high capacity for protein expression, and industrial applicability. $^{30,38}$ These advantages are relevant not only to the simple conversion of codeine from thebaine-rich feedstocks, as demonstrated here, but also potentially for production from simple carbon sources. ${ }^{16} \mathrm{We}$ reconstituted the three-step biosynthetic pathway in a single E. coli strain (for strategy 1) or segregated the pathway into two types of E. coli cells (for strategies 2 and 3) to allow active temporal control of the reaction flux. Previously, E. coli had been used as an effective platform for opiate production, generating $(S)$-reticuline, ${ }^{13}$ thebaine, and hydrocodone. ${ }^{16}$

In strategy 1 , we demonstrated the feasibility of producing codeine from thebaine in a single E. coli strain that expressed both T6ODM and COR enzymes. Thebaine (19\%) was converted to codeine, 10 -fold higher than the $1.8 \%$ thebaine to codeine conversion reported previously using yeast. ${ }^{11} \mathrm{~A}$ major difference between our $E$. coli system and previous yeast systems is the time required for the bioconversion. Typically, yeast is cultured simultaneously with thebaine bioconversion, resulting in a time frame of $\sim 72 \mathrm{~h}^{22}$ We separated $E$. coli growth and the biotransformation processes in this study to allow mass production and storage of the biocatalyst prior to use. A time frame of $45 \mathrm{~min}$ was required for complete conversion from thebaine to codeine, 96-fold shorter than reported in yeast. The reduced reaction time and associated increased volumetric productivity $(0.10 \pm 0.05 \mathrm{~g} /(\mathrm{L} \cdot \mathrm{h}))$ are significant for future industrial deployment of this thebaine to codeine bioconversion process, with the productivity approaching those reported for other industrial productions of fine chemicals by biotransformation (ranging from 1 to $54.6 \mathrm{~g}$ / $(\mathrm{L} \cdot \mathrm{h})$ for 10 reported commercially successful processes) and within the range of productivities reported for other pharmaceutical biotransformations $(0.0002-14 \mathrm{~g} /(\mathrm{L} \cdot \mathrm{h})$ across 17 compounds). ${ }^{27}$

Cell compartmentalization was shown to be a promising route by which the codeine yield could be improved in strategies 2 and 3, which employed E. coli cells with or without the enzyme NISO. In strategy 2 , which uses only two enzymes, thebaine was converted to neopinone by T6ODM present within E. coli cells; the delayed addition of COR-containing cells then provided time for the spontaneous conversion of neopinone to codeinone, allowing an increased yield of codeine at $0.48 \mathrm{mM}$ ( $\Delta t$ of both 45 and $60 \mathrm{~min}$ ), compared with a yield of $0.19 \mathrm{mM}$ in strategy 1 . The codeine/neopine ratio was also improved to 55:45 $(\Delta t=45 \mathrm{~min})$ and 59:41 $(\Delta t$ $=60 \mathrm{~min})$, compared to a ratio of $20: 80$ in strategy 1 . These improvements illustrate the impact of temporal control on the conversion between neopinone and codeinone and therefore on the yield of codeine and associated reduction in neopine yield. This result demonstrates the effectiveness of cell compartmentalization as a strategy for control of the reaction cascade.

Strategy 3 generated the best conditions for codeine production, employing cell compartmentalization together with the enzyme NISO. The introduction of NISO led to $0.64 \mathrm{mM}$ ( $64 \%$ yield) codeine ( $\Delta t$ of both 30 and $45 \mathrm{~min}$ ), with a ratio of codeine/neopine of $70: 30(\Delta t=30 \mathrm{~min})$ or 74:26 $(\Delta t=45 \mathrm{~min})$, further improving both the yield and specificity of codeine production compared to strategy 2 . This set of experiments further demonstrates how the rate of conversion between neopinone and codeinone, improved by NISO catalysis, impacts the yield of codeine produced. This approach also slightly improved volumetric productivity $0.19 \pm$ $0.02 \mathrm{~g} /(\mathrm{L} \cdot \mathrm{h})$ for strategy 3 compared to $0.15 \pm 0.01 \mathrm{~g} /(\mathrm{L} \cdot \mathrm{h})$ for strategy 2.

Our approach extends an earlier concept of enzyme subcellular compartmentalization, where the COR enzyme was localized in a yeast organelle, ${ }^{11}$ when the entire pathway to morphine was integrated in a single yeast cell. In this instance, COR isolation within the organelle increased specificity within the pathway, leading to an increase from 44 to $86 \%$ morphine, through reduced production of the undesired neomorphine; thebaine to morphine conversion, however, was only improved from 0.8 to $1 \% .{ }^{11}$ In the current study, we targeted a semisynthetic approach using thebaine produced by the poppy combined with a three-step biocatalytic cascade, compared to subcellular compartmentalization in a single yeast cell where the product was generated entirely from simple carbon sources. The semisynthetic approach provides a simpler cellular compartmentalization strategy, which allows effective spatial and temporal control over reactions within separate cells and significantly increased both substrate conversion efficiency and volumetric productivity for key steps between thebaine and codeine.

The cell compartmentalization strategy studied here could be further optimized with other complementary approaches. For example, the level of enzyme expression may be manipulated to optimize the enzyme/substrate ratios present within the three-step pathway. Previously, in yeast, a strong promoter was used for NISO expression together with a weak promoter for COR expression to improve codeine production from $\sim 200$ to $\sim 300 \mu \mathrm{g} / \mathrm{L} / \mathrm{OD} .{ }^{10}$ We envisage that faster catalysis may be achieved with higher expression of the NISO enzyme or discovery of improved NISO variants. Increasing NISO activity may further reduce the time delay needed before 
the addition of cells containing COR. Similarly, the expression level of T6ODM and/or COR may be increased, as this strategy (also involving CODM enzyme that catalyzes the conversion of codeine to morphine) increased the yield of morphine from 2 to $5.2 \mathrm{mg} / \mathrm{L} .{ }^{11}$ We predict that the improved expression could either speed up reaction rates or reduce biocatalyst loading-both lead to reduced process costs (less plant time or lower biocatalyst usage). A greater understanding of the kinetic parameters of T6ODM, NISO, and COR, in particular within cells, could also assist in biotransformation design. This kinetic understanding could be coupled with mutagenesis to improve the kinetics of T6ODM and NISO or selectivity of COR, which could further reduce the generation of byproduct neopine.

\section{CONCLUSIONS}

In conclusion, the cellular compartmentalization strategy for $E$. coli proposed in this work dramatically improved thebaine to codeine conversion (on average, $64 \%$ of thebaine was converted to codeine, compared to only $1.8 \%$ reported previously). The strategy also provided high volumetric productivity for codeine manufacture $[0.19 \mathrm{~g} /(\mathrm{L} \cdot \mathrm{h})]$, suggesting compatibility with future industrial application. ${ }^{27}$ The $E$. coli system presented could also be incorporated into or coupled with other microbial biotransformation systems, for example, the reported thebaine production E. coli system, ${ }^{16}$ to provide a basis for complete biosynthesis of codeine from simple carbon sources in microbes. The potential of coculture strategies has been discussed in recent review articles, ${ }^{8,30}$ suggesting that the cellular compartmentalization strategy employed here, using multiple cell types for spatial or temporal control, could also be applied for the biosynthesis of other secondary metabolites.

\section{EXPERIMENTAL SECTION}

Chemicals and Reagents. Antibiotics, analytical grade glycerol, isopropyl $\beta$-d-1-thiogalactopyranoside (IPTG), sodium chloride, sodium ascorbate, glucose and iron sulfate heptahydrate, HPLC grade acetonitrile, methanol, trifluoracetic acid (TFA), dichloromethane (DCM), and acetic acid were purchased from Merck (U.S.A.). Restriction enzymes, T4 DNA ligase, E. coli 5- $\alpha$ competent cells, and E. coli BL21(DE3) competent cells were purchased from New England BioLabs Inc., Australia. Yeast extract and tryptone for medium preparation were purchased from Oxoid, Thermo Scientific, UK. Water for all the experiments was Milli-Q water with a resistivity of $\geq 18.2 \mathrm{M} \Omega \cdot \mathrm{cm}$. Thebaine and codeine were provided by Sun Pharmaceutical Industries Australia Pty Ltd. Neopine was produced by whole cell biotransformation from thebaine (see the Whole Cell Biotransformation section) following a previously reported protocol. Briefly, the final biotransformation product was centrifuged at $3200 \mathrm{~g}$ for 15 min. The supernatant was concentrated five times and then purified by preparative scale reverse-phase HPLC (RP-HPLC) with a linear gradient of 5-28\% buffer B and a flow rate of 20 $\mathrm{mL} / \mathrm{min}$ over $20 \mathrm{~min}$ at $25{ }^{\circ} \mathrm{C}$ (buffer A: $0.1 \%$ TFA in water; buffer B: $0.1 \%$ TFA in methanol). Fractions were collected based on peak and combined for freeze-drying. DCM $(7.5 \mathrm{~mL})$ was added to the freeze-dried residue; phosphate buffer (2.5 $\mathrm{mL}, 0.5 \mathrm{M}, \mathrm{pH} 8.0$ ) was used to wash the residue, which was washed repeatedly thrice; DCM was evaporated with nitrogen blow; and the residue was then dissolved in $10 \%$ acetic acid, followed by nine parts of Milli-Q water and freeze-dried again. The final product was confirmed using NMR spectroscopy (see Figure S1 and Table S1), mass spectrometry (MS), and RP-HPLC for its structure, mass, and purity. The manipulation on all the schedule 8 poisons complies with the appropriate Government Act.

Plasmids Used in This Study. Four plasmids were used in this work for biotransformation: pETDeut-1-PsT6ODMPbCOR1-1 (P1), pACYC-PsT6ODM (P2), pET28aPbCOR1-1 (P3), and pETDuet-1-PsT6ODM-PsNISO (P4). The maps for each plasmid are shown in Figures S3-S6. Two plasmids (P2 and P3) were provided by Sun Pharmaceutical Industries Australia Pty Ltd. Plasmid P2 was constructed by ligating thebaine 6-O-demethylase from $P$. somniferum (PsT6ODM, GenBank accession number GQ500139.1) into the pACYC vector. Plasmid P3 was constructed by ligating codeine reductase variant $1-1$ from $P$. bracteatum (PbCOR1-1, GenBank accession number ACM44064.1) into the pET28a vector. Plasmid P1 was constructed based on a plasmid (pETDeut1-PsT6ODM-PsCOR1-5, P5) provided by Sun Pharmaceutical Industries Australia Pty Ltd. Plasmid P5 was digested with restriction enzymes NdeI and XhoI to remove the PsCOR1-5 DNA fragment and provide a vector backbone with sticky ends. Plasmid P3 was digested with the same enzymes to provide the insert DNA PbCOR1-1. The insert and backbone were ligated with the T4 DNA ligase to form plasmid P1. Plasmid P4 was constructed based on a plasmid pUCIDT-PsNISO (P6) custom synthesized by Integrated DNA Technologies, Inc. NISO from P. somniferum (PsNISO, GenBank accession number QBG82386.1) was cloned into the IDT vector pUCIDT to form plasmid P6. PsNISO DNA was cut out with NdeI and XhoI as an insert and ligated to plasmid $\mathrm{P} 5$ vector backbone to construct plasmid P4. All the T6ODM, COR, and NISO DNA sequences were synthesized with codon optimization in order to improve protein translation in E. coli while leaving the amino acid sequences of the final protein products unchanged compared to the wild type. DNA sequence of maltose binding protein was added to the T6ODM sequence to improve the solubility of T6ODM. The codon optimized DNA sequences are shown in Figure S7. All plasmids were transformed into E. coli 5- $\alpha$ competent cells for the purpose of cloning and DNA sequencing prior to the transformation of E. coli BL21(DE3) competent cells for enzyme expression and biotransformation.

E. coli Strains Used in This Study. Four types of E. coli strains were constructed to express enzymes for whole cell biotransformation by transforming E. coli BL21(DE3) competent cells with the four above-mentioned plasmids (P1-P4) individually. The transformed cells were named as T6ODM/COR cell, T6ODM cell, COR cell, and T6ODM/ NISO cell, corresponding to the cells transformed with P1, P2, P3, and P4 plasmids. They were used either independently or in combination for the three different biotransformation strategies. Specifically, strategy 1 used the single-cell strain of the T6ODM/COR cell, which was expected to convert thebaine to codeine within the cell; strategy 2 used a mixture of T6ODM cell and COR cell, in which thebaine was expected to convert to neopinone in the T6ODM cell, simultaneously equilibrated to codeinone and finally reduced to codeine in the COR cell; strategy 3 used a mixture of T6ODM/NISO cell and COR cell, in which thebaine was expected to convert to codeinone in the T6ODM/NISO cell and further catalyzed to codeine in COR cell. 
Bacterial Cell Cultivation and Protein Expression. Cells were cultured overnight at $37{ }^{\circ} \mathrm{C}$ in $\mathrm{LB}$ medium containing corresponding antibiotics. One milliliter of the overnight cell culture was inoculated into a conical flask that contained $50 \mathrm{~mL}$ of 2-YT medium, corresponding antibiotics, and $1 \mathrm{~mL}$ of glycerol. IPTG $(0.1 \mathrm{mM})$ was added to the culture when $\mathrm{OD}_{600}$ reached $0.4-0.8$ to start protein expression, and the expression was continued for $22 \mathrm{~h}$ at 18 ${ }^{\circ} \mathrm{C}$. Cells pellets were collected by centrifugation at $3200 \mathrm{~g}$ for $15 \mathrm{~min}$ and resuspended in $15 \%$ glycerol to obtain a cell solution of $\mathrm{OD}_{600}$ at 100 for biotransformation.

Whole Cell Biotransformation. Fresh E. coli cells $\left(\mathrm{OD}_{600}\right.$ $=100$ ) were added into the biotransformation mixture with a final cell concentration of $\mathrm{OD}_{600}$ at 10 . For strategy 2 and strategy 3 that used two types of cell strains, each strain had a final concentration of $\mathrm{OD}_{600}$ at 10 in the reaction. The substrate, buffer, and cofactors in a $20 \mathrm{~mL}$ reaction mixture consisted of $1 \mathrm{mM}$ thebaine, $100 \mathrm{mM}$ phosphate buffer ( $\mathrm{pH}$ at $6.0), 0.5 \% \mathrm{w} / \mathrm{w}$ glucose, $10 \mu \mathrm{M} \mathrm{FeSO}_{4}$, and $10 \mathrm{mM}$ sodium ascorbate. Biotransformation was incubated at $24{ }^{\circ} \mathrm{C}$ and stirred at $220 \mathrm{rpm}$. Samples were taken regularly at different time intervals. Samples collected were centrifuged at $16,000 \mathrm{~g}$ $\mathrm{rpm}$ for $10 \mathrm{~min}$, and the supernatant was analyzed by LC-MS for alkaloid quantification.

LC-MS Analysis of Alkaloids. Detection of alkaloids was performed using a Shimadzu LCMS-2020 liquid chromatograph mass spectrometer. Analysis was carried out on an Onyx Monolithic C18 column $(100 \times 4.6 \mathrm{~mm}$, Phenomenex Australia Pty Ltd.), with a linear gradient of $0-20 \%$ buffer B and at a flow rate increased from 1 to $2.5 \mathrm{~mL} / \mathrm{min}$ over $10 \mathrm{~min}$ at $25{ }^{\circ} \mathrm{C}$ (buffer A: $0.1 \%$ TFA in water; buffer B: $0.1 \%$ TFA in acetonitrile). Detector wavelength was set up at $285 \mathrm{~nm}$ with a reference wavelength set at $360 \mathrm{~nm}$. Alkaloid compounds in biotransformation samples were identified by comparing to alkaloid standards, referring to both RT (neopine at $7.3 \mathrm{~min}$, codeine at $8.1 \mathrm{~min}$, thebaine at $11.4 \mathrm{~min}$ ) and mass to charge ratio $(m / z)$ (neopine $m / z=300$, codeine $m / z=300$, thebaine $m / z=312$ ). Shimadzu LabSolutions software was used to integrate the peak area for each compound to quantify the concentration of alkaloid in each sample by referring to the peak area of alkaloid standard series with concentration ranging from 67 to $333 \mu \mathrm{g} / \mathrm{mL}$. Both sample and standard solutions were injected into the LC-MS column with a $10 \mu \mathrm{L}$ injection volume for analysis.

\section{ASSOCIATED CONTENT}

\section{SI Supporting Information}

The Supporting Information is available free of charge at https://pubs.acs.org/doi/10.1021/acsomega.0c00282.

Molecular structure of neopine and ${ }^{1} \mathrm{H}$ NMR spectrum of neopine; ${ }^{1} \mathrm{H}$ NMR multiplicity analysis of neopine in comparison with reported multiplicity; comparison of amino acid sequences for COR from different species; construction of the pETDeut-1-PsT6ODM-PbCOR1-1 (P1) including both the PsT6ODM gene and the $P b C O R 1-1$ gene used in this study; construction of the pACY-PsT6ODM (P2) including the PsT6ODM gene used in this study; construction of the pET28aPbCOR1-1 (P3) including the PbCOR1-1 gene used in this study; construction of the pETDeut-1-PsT6ODMPsNISO including both the PsT6ODM gene and the PsNISO gene used in this study; sequences of the
PsT6ODM gene, PbCOR1-1 gene, and PsNISO gene with the $E$. coli codon optimization used in this study; and time required to fully consume thebaine and generate final products of both codeine and neopine (PDF)

\section{AUTHOR INFORMATION}

\section{Corresponding Author}

Sally L. Gras - Department of Chemical Engineering and The Bio21 Molecular Science and Biotechnology Institute, The University of Melbourne, Melbourne, Victoria 3010, Australia; 다이.org/0000-0002-4660-1245; Email: sgras@ unimelb.edu.au

\section{Authors}

Xu Li - Department of Chemical Engineering and The Bio21 Molecular Science and Biotechnology Institute, The University of Melbourne, Melbourne, Victoria 3010, Australia; 이이.org/ 0000-0003-3317-9257

Konrad Krysiak-Baltyn - Department of Chemical Engineering and The Bio21 Molecular Science and Biotechnology Institute, The University of Melbourne, Melbourne, Victoria 3010, Australia

Luke Richards - Department of Chemical Engineering and The Bio21 Molecular Science and Biotechnology Institute, The University of Melbourne, Melbourne, Victoria 3010, Australia; (1) orcid.org/0000-0002-7447-1599

Ailsa Jarrold - Sun Pharmaceutical Industries Australia Pty Ltd., Port Fairy, Victoria 3281, Australia; 이이.org/00000001-5494-0901

Geoffrey W. Stevens - Department of Chemical Engineering, The University of Melbourne, Melbourne, Victoria 3010, Australia; (1) orcid.org/0000-0002-5788-4682

Tim Bowser - Impact Science Consulting, Melbourne, Victoria 3081, Australia

Robert E. Speight - School of Biology and Environmental Science, Queensland University of Technology, Brisbane, Queensland 4000, Australia; (1) orcid.org/0000-0003-41618272

Complete contact information is available at:

https://pubs.acs.org/10.1021/acsomega.0c00282

\section{Notes}

The authors declare no competing financial interest.

\section{ACKNOWLEDGMENTS}

This work was supported by The Australian Research Council Linkage Project scheme (LP160100182) and Sun Pharmaceutical Industries Australia Pty Ltd.. We thank Dr Angela Bridges and her team at GSK, Stevenage, UK, for advice and provision of plasmids; The Bio21 Melbourne Mass Spectrometry and Proteomics platform and Melbourne Magnetic Resonance platform at The University of Melbourne for technical support; and Zongjia Chen, Garrick Spencer, and Dr Shuai Nie at The University of Melbourne for discussion and technical support.

\section{REFERENCES}

(1) Symons, G. M.; Hudson, C. J.; Livermore, M. L. Papaver somniferum with high concentration of codeine. WO $2009143574 \mathrm{Al}$, 2009.

(2) Winzer, T.; Walker, T. C.; MEADE, F.; Larson, T. R.; Graham, I.A. Modified Plant. WO 2017122011 A1, 2017. 
(3) Department of Justice, US Drug Enforcement Administration. https://www.dea.gov/press-releases/2018/08/16/justicedepartment-dea-propose-significant-opioid-manufacturing-reduction (accessed 2020-01-10).

(4) Fist, A. J. The Tasmanian Poppy Industry: A Case Study of the Application of Science and Technology. Proceedings of the Australian Agronomy Conference 2001.

(5) WHO Model List of Essential Medicines, 21st List; World Health Organization, 2019 (https://www.who.int/medicines/publications/ essentialmedicines/en/).

(6) Estimated World Requirements for 2019-Statistics for 2017, INCB Technical Report (https://www.incb.org/incb/en/narcoticdrugs/Technical_Reports/narcotic_drugs_reports.html).

(7) Galanie, S.; Thodey, K.; Trenchard, I. J.; Filsinger Interrante, M.; Smolke, C. D. Complete biosynthesis of opioids in yeast. Science 2015, 349, 1095-1100.

(8) Li, S.; Li, Y.; Smolke, C. D. Strategies for microbial synthesis of high-value phytochemicals. Nat. Chem. 2018, 10, 395-404.

(9) Hagel, J. M.; Facchini, P. J. Dioxygenases catalyze the Odemethylation steps of morphine biosynthesis in opium poppy. Nat. Chem. Biol. 2010, 6, 273.

(10) Dastmalchi, M.; Chen, X.; Hagel, J. M.; Chang, L.; Chen, R.; Ramasamy, S.; Yeaman, S.; Facchini, P. J. Neopinone isomerase is involved in codeine and morphine biosynthesis in opium poppy. Nat. Chem. Biol. 2019, 15, 384-390.

(11) Thodey, K.; Galanie, S.; Smolke, C. D. A microbial biomanufacturing platform for natural and semisynthetic opioids. Nat. Chem. Biol. 2014, 10, 837.

(12) Ehrenworth, A. M.; Peralta-Yahya, P. Accelerating the semisynthesis of alkaloid-based drugs through metabolic engineering. Nat. Chem. Biol. 2017, 13, 249.

(13) Nakagawa, A.; Minami, H.; Kim, J.-S.; Koyanagi, T.; Katayama, T.; Sato, F.; Kumagai, H. A bacterial platform for fermentative production of plant alkaloids. Nat. Commun. 2011, 2, 326.

(14) Fossati, E.; Ekins, A.; Narcross, L.; Zhu, Y.; Falgueyret, J.-P.; Beaudoin, G. A. W.; Facchini, P. J.; Martin, V. J. J. Reconstitution of a 10-gene pathway for synthesis of the plant alkaloid dihydrosanguinarine in Saccharomyces cerevisiae. Nat. Commun. 2014, 5, 3283.

(15) Li, Y.; Smolke, C. D. Engineering biosynthesis of the anticancer alkaloid noscapine in yeast. Nat. Commun. 2016, 7, 12137.

(16) Nakagawa, A.; Matsumura, E.; Koyanagi, T.; Katayama, T.; Kawano, N.; Yoshimatsu, K.; Yamamoto, K.; Kumagai, H.; Sato, F.; Minami, H. Total biosynthesis of opiates by stepwise fermentation using engineered Escherichia coli. Nat. Commun. 2016, 7, 10390

(17) Winzer, T.; Gazda, V.; He, Z.; Kaminski, F.; Kern, M.; Larson, T. R.; Li, Y.; Meade, F.; Teodor, R.; Vaistij, F. E.; Walker, C.; Bowser, T. A.; Graham, I. A. A Papaver somniferum 10-Gene Cluster for Synthesis of the Anticancer Alkaloid Noscapine. Science 2012, 336, 1704-1708.

(18) Winzer, T.; Kern, M.; King, A. J.; Larson, T. R.; Teodor, R. I.; Donninger, S. L.; Li, Y.; Dowle, A. A.; Cartwright, J.; Bates, R.; Ashford, D.; Thomas, J.; Walker, C.; Bowser, T. A.; Graham, I. A. Morphinan biosynthesis in opium poppy requires a $\mathrm{P} 450$-oxidoreductase fusion protein. Science 2015, 349, 309-312.

(19) Li, Y.; Li, S.; Thodey, K.; Trenchard, I.; Cravens, A.; Smolke, C. D. Complete biosynthesis of noscapine and halogenated alkaloids in yeast. Proc. Natl. Acad. Sci. U.S.A. 2018, 115, E3922-E3931.

(20) Ro, D.-K.; Paradise, E. M.; Ouellet, M.; Fisher, K. J.; Newman, K. L.; Ndungu, J. M.; Ho, K. A.; Eachus, R. A.; Ham, T. S.; Kirby, J.; Chang, M. C. Y.; Withers, S. T.; Shiba, Y.; Sarpong, R.; Keasling, J. D. Production of the antimalarial drug precursor artemisinic acid in engineered yeast. Nature 2006, 440, 940-943.

(21) Gollwitzer, J.; Lenz, R.; Hampp, N.; Zenk, M. H. The transformation of neopinone to codeinone in morphine biosynthesis proceeds non-enzymatically. Tetrahedron Lett. 1993, 34, 5703-5706.

(22) Dastmalchi, M.; Chang, L.; Torres, M. A.; Ng, K. K. S.; Facchini, P. J. Codeinone reductase isoforms with differential stability, efficiency and product selectivity in opium poppy. Plant J. 2018, 95, 631-647.
(23) Tam, W. H. J.; Kurz, W. G. W.; Constabel, F.; Chatson, K. B. Biotransformation of thebaine by cell suspension cultures of Papaver somniferum cv. Marianne. Phytochemistry 1982, 21, 253-255.

(24) Parker, H. I.; Blaschke, G.; Rapoport, H. Biosynthetic Conversion of Thebaine to Codeine. J. Am. Chem. Soc. 1972, 94, $1276-1282$.

(25) Kuiper, C.; Vissers, M. C. M. Ascorbate as a co-factor for feand 2-oxoglutarate dependent dioxygenases: physiological activity in tumor growth and progression. Front. Oncol. 2014, 4, 359.

(26) Lenz, R.; Zenk, M. H. Purification and Properties of Codeinone Reductase (NADPH) from Papaver somniferum Cell Cultures and Differentiated Plants. Eur. J. Biochem. 1995, 233, 132-139.

(27) Straathof, A. J. J.; Panke, S.; Schmid, A. The production of fine chemicals by biotransformations. Curr. Opin. Biotechnol. 2002, 13, $548-556$.

(28) Lee, E. J.; Hwang, I. K.; Kim, N. Y.; Lee, K. L.; Han, M. S.; Lee, Y. H.; Kim, M. Y.; Yang, M. S. An Assessment of the Utility of Universal and Specific Genetic Markers for Opium Poppy Identification. J. Forensic Sci. 2010, 55, 1202-1208.

(29) Unterlinner, B.; Lenz, R.; Kutchan, T. M. Molecular cloning and functional expression of codeinone reductase: the penultimate enzyme in morphine biosynthesis in the opium poppy Papaver somniferum. Plant J. 1999, 18, 465-475.

(30) Pyne, M. E.; Narcross, L.; Martin, V. J. J. Engineering Plant Secondary Metabolism in Microbial Systems. Plant Physiol. 2019, 179, 844-861.

(31) Spaans, S. K.; Weusthuis, R. A.; van der Oost, J.; Kengen, S. W. M. NADPH-generating systems in bacteria and archaea. Front. Microbiol. 2015, 6, 742.

(32) Chemler, J. A.; Fowler, Z. L.; McHugh, K. P.; Koffas, M. A. G. Improving NADPH availability for natural product biosynthesis in Escherichia coli by metabolic engineering. Metab. Eng. 2010, 12, 96104.

(33) Slonczewski, J. L.; Fujisawa, M.; Dopson, M.; Krulwich, T. A. Cytoplasmic $\mathrm{pH}$ Measurement and Homeostasis in Bacteria and Archaea. Adv. Microb. Physiol. 2009, 55, 1-317.

(34) Winkel, B. S. J. Metabolic Channeling in Plants. Annu. Rev. Plant Biol. 2004, 55, 85-107.

(35) Lundemo, M. T.; Woodley, J. M. Guidelines for development and implementation of biocatalytic P450 processes. Appl. Microbiol. Biotechnol. 2015, 99, 2465-2483.

(36) Akhtar, M. K.; Jones, P. R. Construction of a synthetic YdbKdependent pyruvate:H2 pathway in Escherichia coli BL21(DE3). Metab. Eng. 2009, 11, 139-147.

(37) Sonderegger, M.; Sauer, U. Evolutionary engineering of Saccharomyces cerevisiae for anaerobic growth on xylose. Appl. Environ. Microbiol. 2003, 69, 1990-1998.

(38) Baneyx, F. Recombinant protein expression in Escherichia coli. Curr. Opin. Biotechnol. 1999, 10, 411-421. 